\title{
Molecular Line Observations of the Protoplanetary Nebula, IRAS 19312+1950
}

\author{
Shuji Deguchi \\ Nobeyama Radio Observatory, National Astronomical Observatory, \\ Minamimaki, Minamisaku, Nagano 384-1305, Japan
}

Jun-ichi Nakashima

Graduate University for Advanced Studies, Nobeyama Radio Observatory, National Astronomical Observatory, Minamimaki, Minamisaku, Nagano 384-1305, Japan

\begin{abstract}
We give a summary of radio molecular-line and infrared imaging observations of the newly found O-rich protoplanetary nebula, IRAS $19312+1950$. This object exhibits a bipolar nebulosity of size $\sim 30^{\prime \prime}$ on the near-IR $(J H K)$ images. A ring-like structure with a diameter of about $10^{\prime \prime}$ is seen surrounding the central star. Toward this object, we detected thermal lines of $\mathrm{CO}, \mathrm{HCO}^{+}, \mathrm{SO}$, and $\mathrm{SiO}$ (O-bearing) molecules and $\mathrm{CN}, \mathrm{CS}, \mathrm{HNC}, \mathrm{NH}_{3}, \mathrm{~N}_{2} \mathrm{H}^{+}$, and $\mathrm{HC}_{3} \mathrm{~N}$ (C- and N-bearing) molecules, as well as $\mathrm{H}_{2} \mathrm{O}$ and $\mathrm{SiO}$ masers lines. The line profiles of ${ }^{12} \mathrm{CO}, \mathrm{HCN}$, $\mathrm{SO}$, and $\mathrm{HCO}^{+}$are composed of broad $\left(\Delta v \sim 30-40 \mathrm{~km} \mathrm{~s}^{-1}\right)$ and narrow $\left(\Delta v<5 \mathrm{~km} \mathrm{~s}^{-1}\right)$ components. The $\mathrm{SiO}$ profile $(J=2-1 v=0$ thermal line) has only broad component, and the line profiles of non-O-bearing molecules (except HCN) have only a narrow component. Both components are spatially strongly peaked in intensity at the center of this object within $\mathrm{a} \sim 15^{\prime \prime}$ telescope beam. We infer that both components originate from cool material surrounding the central star. This object is most likely an O-rich protoplanetary nebula with high mass loss rate and envelope chemistry similar to that of $\mathrm{OH} 231.8+0.4$.
\end{abstract}

\section{Introduction}

In the course of $\mathrm{SiO}$ maser survey of cold IRAS objects, we found $\mathrm{SiO}$ maser emission from IRAS 19312+1950 (Nakashima \& Deguchi 2000). This object exhibits an IRAS color of protoplanetary nebulae, $C_{12}=0.47 ; C_{12}$ is the logarithmic ratio of the IRAS $25 \mu \mathrm{m}$ to $12 \mu \mathrm{m}$ flux density. The 2MASS $J H K$ images revealed a bipolar nebulosity of size about $30^{\prime \prime}$ (Nakashima \& Deguchi 2000). Triple spurs seen on the near-infrared images are apparently extended to the south-west from the central star, indicating the direction of the bipolar axis to be at P.A. $\sim 45$ degree from the north. Because $\mathrm{H}^{13} \mathrm{CN}$ and $\mathrm{SO}$ lines were also detected simultaneously with the $\mathrm{SiO} J=2-1 v=1$ maser line, it was expected that a number of other molecular lines can be seen toward this object. 

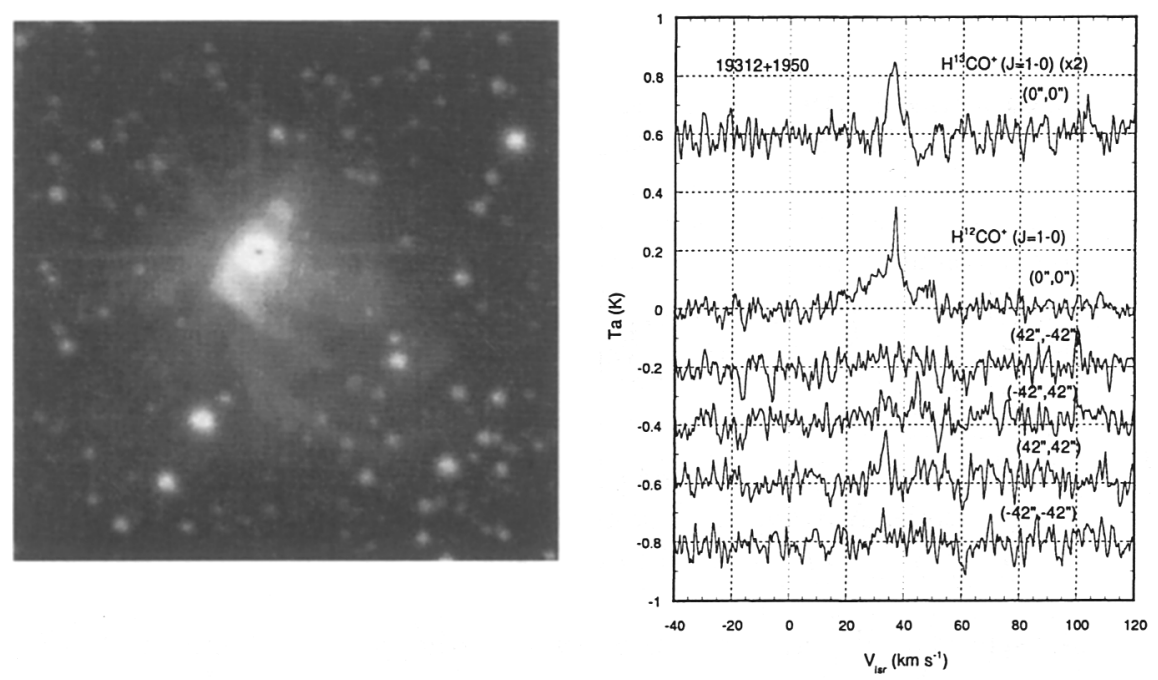

Figure 1. Left panel shows a $J H K$ color composite of IRAS $19312+1950$ taken by the UH $2.2 \mathrm{~m}$ telescope. The image size is $65^{\prime \prime} \times 65^{\prime \prime}$, and the north is up, and the east is left (with courtesy of M. Tamura). Right panel shows spectra of $\mathrm{H}^{13} \mathrm{CO}^{+}$and $\mathrm{H}^{12} \mathrm{CO}^{+}$ $J=1-0$ thermal lines. The $\mathrm{H}^{12} \mathrm{CO}^{+}$line exhibits broad and narrow components, while the $\mathrm{H}^{13} \mathrm{CO}^{+}$line exhibits only the narrow component. The bottom 4 spectra are taken at the positions offset by one arcminute from the object. Both the narrow and broad components are strongly peaked at the object position.

In order to investigate this object further, we have made radio and nearinfrared observations. In this paper, we report some of the new results from these observations.

\section{Observations and results}

\subsection{Infrared Observations}

Near-infrared $(J H K)$ imaging observations of this objects were made in 2000 July using the ANU (Australian National University) 2.3-m telescope at SidingSpring observatory with the CASPIR array camera. Additional imaging observations were made in 2000 October using UH 2.2-m telescope (University of Hawaii) at Mauna Kea with the SIRIUS array camera. (see Nagayama et al. 2000) and using SUBARU 8.3-m telescope (National Astronomical Observatory, Japan) with the CIAO array camera. The $J H K$ color composite image taken with the UH 2.2-m telescope is shown on the left panel of Figure 1. The nebula has three conspicuous spurs which are extended to the south west, and the ring-like structure which is elongated perpendicularly to the direction of spurs. Because of this shape, we call this object as "Jellyfish Nebula". The size of the ring structure is about $10^{\prime \prime}$ in diameter and it is clearly resolved on the SUBARU 

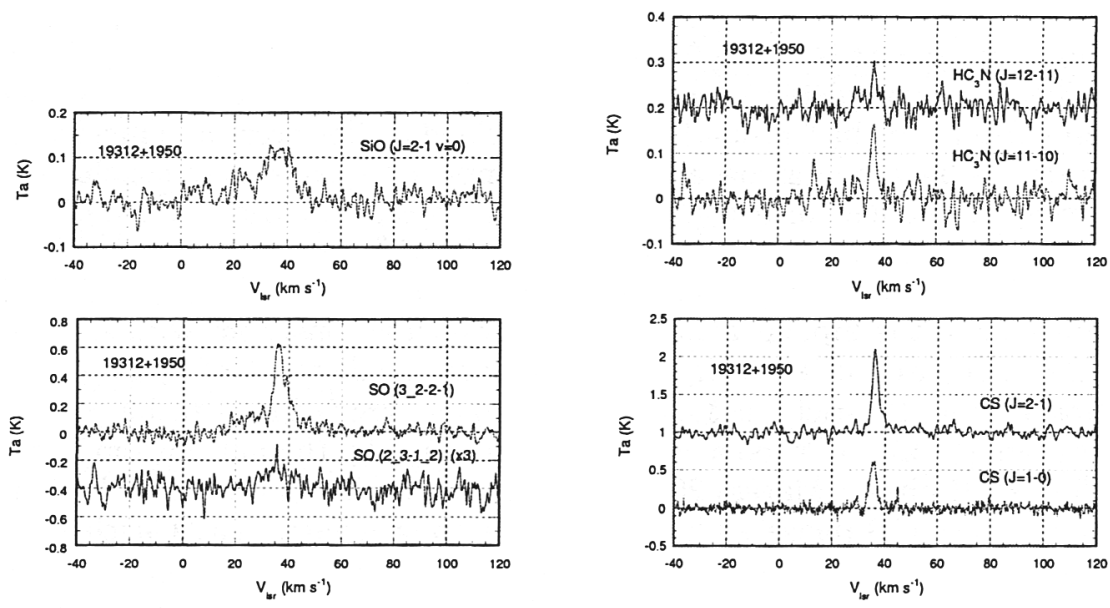

Figure 2. Left panels show spectra of the $\mathrm{SiO} J=1-0 v=0$ thermal line and the SO $3_{2}-2_{1}$ and $2_{3}-1_{2}$ thermal lines. The SiO line exhibits the broad component, only but the SO lines exhibit both the broad and narrow components. Right panels show the spectra of the $\mathrm{HC}_{3} \mathrm{~N}$ $J=12-11$ and $J=11-10$ lines, and the CS $J=2-1$ and $J=1-0$ lines. They have only the narrow component.

$J H K$ images. Magnitude of the central star is $K \sim 6.2$. We also detected $\mathrm{H}_{2}$ $2.112 \mu \mathrm{m}$ emission $10^{\prime \prime}$ north of the central star with the ANU $2.3 \mathrm{~m}$ telecope.

\subsection{Radio Observations}

Radio molecular-line observations were made in 2001 February using the Nobeyama 45-m telescope. We detected thermal emission of ${ }^{12} \mathrm{CO},{ }^{13} \mathrm{CO}, \mathrm{C}^{18} \mathrm{O}$, $\mathrm{SiO}, \mathrm{SO}, \mathrm{CS}, \mathrm{HCN}, \mathrm{HC}^{13} \mathrm{~N}, \mathrm{HC}_{3} \mathrm{~N}, \mathrm{HCO}^{+}$, and $\mathrm{N}_{2} \mathrm{H}^{+}$toward this object. Additional continuum observations were made at Nobeyama. Continuum emission of $0.1 \mathrm{Jy}$ at 5 sigma was detected at $150 \mathrm{GHz}$; this is consistent with the model with $\dot{M}=2 \times 10^{-4} M_{\odot} y r^{-1}$ at $D=3 \mathrm{kpc}$ (Knapp et al. 1993).

The line profiles of O-bearing molecules exhibit both broad $\left(\sim 40 \mathrm{~km} \mathrm{~s}^{-1}\right)$ and narrow ( $\lesssim 5 \mathrm{~km} \mathrm{~s}^{-1}$ ) components (left panel of figure 2), though the profiles of $\mathrm{C}$ - and N-bearing molecules have only narrow components (right panel of figure 2). We also mapped the source in the $J=1-0$ lines of ${ }^{12} \mathrm{CO},{ }^{13} \mathrm{CO}, \mathrm{C}^{18} \mathrm{O}$, $\mathrm{HCN}, \mathrm{HCO}^{+}$and confirmed that line intensities of both components are strongly peaked at the source position within about $15^{\prime \prime}$ telescope beam. It is curious that no $\mathrm{SO}_{2}$ lines were detected from this object, whereas both $\mathrm{SO}$ and $\mathrm{SO}_{2}$ lines have been observed in a number of O-rich circumstellar envelopes (Guilloteau et al. 1986). An example for a mixture of C- and O-rich chemistries was found previously in OH 231.8+4.2 (Deguchi \& Goldsmith 1985; Morris et al. 1987). 


\subsection{Structure of the nebula}

The broad component is surely formed in the expanding envelope of this object. It is difficult to completely deny the possibility that the narrow component originates from fore/background molecular clouds. However, a coincidence of peak positions between narrow and broad components leads us to conclude that the narrow component also comes from this object. In the ${ }^{12,13} \mathrm{CO} J=1-$ 0 maps, the object is weakly resolved with the $15^{\prime \prime}$ beam. In the ${ }^{13} \mathrm{CO}$ line, we detected a small velocity gradient $\left(\sim 0.6 \mathrm{~km} \mathrm{~s}^{-1}\right.$ per $\left.7.5^{\prime \prime}\right)$ in the direction perpendicular to the bipolar axis (NE-SW), which is consistent with a rotation of the disk/ring around a $\sim 9 M_{\odot}$ central star at the distance of $3 \mathrm{kpc}$.

Progenitors of O-rich protoplanetary nebulae are inferred to be relatively massive stars $\left(Z 4 M_{\odot}\right)$. Atoms as ${ }^{12} \mathrm{C}$ are consumed by the hot bottom burning at the TP AGB phase (Marigo 2001) and the massive stars remain to be O-rich throughout the AGB phase with experiencing no or a very short C-rich era.

The origin of the ring-like structure is not clear at this moment. Possibly, the ring is the material which was ejected by the central star in the past and trapped by a binary counterpart. A similar phenomena is observed as silicate carbon stars due to trapped silicate dust around carbon stars (Lloyd-Evans 1990). Alternatively, it is possible to consider that the material formed at the time of star formation ("protoplanet" dust disk) remains until the AGB phase (Melnick et al. 2001). If the narrow component is associated with the ring, the molecular abundance of the ring may provide a clue to resolve this issue.

\section{Conclusion}

We have found an O-rich bipolar nebula, IRAS 19312+1950, which has SiO masers and a ring-like structure. Near-infrared imaging observations revealed the nebulosity of size about $30^{\prime \prime}$ extended to the south west and a ring of about $10^{\prime \prime}$ diameter. The rich molecular lines detected at radio frequencies toward this object imply that the circumstellar chemistry is quite complex in this object.

We thank Peter Wood, Motohide Tamura, and Nario Kuno for their assistance of near-infrared and radio-continuum observations.

\section{References}

Deguchi S., \& Goldsmith, P.F. 1985, Nature, 317, 336

Guilloteau, S., Lucas, R., Nguyen-Q-Rieu, Omont, A. 1986, A\&A, 165, L1

Knapp, G. R., Sandell, G., Robson, E. I. 1993, ApJS, 88, 173

Lloyd-Evans, J. 1990, MN, 243, 336

Melnick, G. J., Neufeld, D. A., Ford, K.E. S., Hollenbach, D. J., Ashby, M. L.N. 2001, Nature, 412,160

Morris, M., Guilloteau, S., Lucas, R., Omont, A. 1987, ApJ, 321, 888

Nakashima, J. \& Deguchi, S. 2000, PASJ 52, L43

Nagayama et al. 2000, Proceeding of "Star Formation 1999", P395 